\title{
Toxocariasis: manifestaciones clínicas y de laboratorio en niños asistidos en un prestador integral de salud privado de Montevideo, Uruguay (2014-2018)
}

Patricia Barrios Godoy, Joaquín Mauvezin†, Yester Basmadjian‡, Beatriz Sayagués§, Gustavo Giachetto"

\section{Resumen}

Introducción: la toxocariasis humana es una parasitosis de amplia distribución en el mundo cuyos agentes etiológicos son Toxocara canis y, en menor medida, Toxocara cati.

Objetivo: describir las características clínicas y paraclínicas de toxocariasis en menores de 15 años asistidos en un prestador integral privado de Montevideo entre 2014 y 2018.

Material y método: estudio descriptivo, retrospectivo, de los menores de 15 años derivados a la policlínica de infectología entre el 1 de enero de 2014 y el 31 de diciembre de 2018 con diagnóstico de toxocariasis confirmada por ELISA. Variables: edad, sexo, motivo de consulta, manifestaciones clínicas, recuento leucocitario y de eosinófilos, parasitosis asociadas, tratamiento y evolución.

Resultados: fueron asistidos 20 niños de los cuales 11 eran asintomáticos, cinco presentaron síndrome de larva migrans visceral (LMV) y cuatro síndrome de larva migrans ocular (LO). Veinte niños recibieron albendazol y uno, además, mebendazol. Aquellos con compromiso ocular recibieron también corticoides vía oral. Se constató pérdida de agudeza visual en dos pacientes.

Discusión y conclusiones: la mayorías de los niños eran asintomáticos, la cuarta parte consultaron por alteraciones visuales cuyas secuelas representan un grave problema de salud potencialmente prevenible. Debería realizarse la búsqueda de la infección mediante serología en niños que presenten eosinofilia con y sin síntomas respiratorios o disminución de la agudeza visual causada por lesión intraocular y en niños con factores de riesgo para esta parasitosis.

Palabras clave: Toxocariasis

Key words: $\quad$ Toxocariasis

* Profesora Agregada de Pediatría. Departamento de Pediatría y Especialidades. Facultad de Medicina, Universidad de la República. Pediatra de la Policlínica de Infectología de Médica Uruguaya. Uruguay.

† Asistente de Pediatría. Departamento de Pediatría y Especialidades. Facultad de Medicina, Universidad de la República. Pediatra Médica Uruguaya. Uruguay.

‡ Profesora del Departamento de Parasitología y Micología. Facultad de Medicina, Universidad de la República. Uruguay.

$\S$ Pediatra. Jefa del Centro Intensivo Neonatal y Pediátrico de Médica Uruguaya. Uruguay.

II Profesor de Clínica Pediátrica. Departamento de Pediatría y Especialidades. Facultad de Medicina, Universidad de la República. Consultante de Infectología de Médica Uruguaya. Uruguay.

Correspondencia: Dra. Patricia Barrios. Clínica Pediátrica "C". Centro Hospitalario Pereira Rossell. Facultad de Medicina, Universidad de la Repú blica, Montevideo. Uruguay. Correo electrónico: patriciabarrios77@gmail.com

Este trabajo cuenta con la aprobación del Comité de Ética de la mutualista Médica Uruguaya.

Los autores declaran no tener conflicto de intereses.

Recibido: 27/2/19

Aprobado: 23/10/19 


\section{Introducción}

La toxocariasis es una zoonosis parasitaria típica de países tropicales y subtropicales causada por especies del género Toxocara, que incluye más de 30 especies, siendo importantes para el ser humano T. canis, y, en menor medida, T. cati, parásitos intestinales de perros y gatos. El hombre es un huésped accidental. Se transmite al ingerir huevos fértiles larvados del parásito con la posterior migración tesidual de larvas liberadas en el intestino $^{(1-6)}$.

Los huevos pueden permanecer varios años en suelos húmedos y soportan la desecación. En niños y adolescentes es frecuente la ingestión accidental de los huevos a través de las manos contaminadas, ya que debido a su actividad lúdica tienen contacto frecuente con áreas de tierra contaminada, tanto en parques como en sus hogares. Hábitos como onicofagia, geofagia y el consumo de vegetales y frutas crudas o mal lavadas favorecen la infección, así como el estrecho contacto con los animales ${ }^{(6-8)}$.

Tras la ingesta de los huevos, estos eclosionan y las larvas penetran la pared intestinal e ingresan a la circulación sistémica con migración a órganos como hígado, pulmones, cerebro u ojos. Dicha migración se acompaña de hemorragia, necrosis e inflamación, con predominio de eosinófilos. En función de la respuesta inmune del huésped, las larvas pueden migrar durante meses o años; permanecer años en estado quiescente encapsuladas en granulomas, o ser destruidas por medio de la respuesta celular ${ }^{(2,3,9)}$.

Se describen dos formas clínicas principales: el síndrome de larva migrans visceral (LMV) y el síndrome de larva migrans ocular (LO) o toxocariasis ocular. Otras formas clínicas incluyen toxocariasis encubierta, neurotoxocariasis y asmatiforme. Una de las secuelas más graves de esta parasitosis es la pérdida de la visión del ojo lesionado. Con respecto a la técnica diagnóstica se recomienda la técnica de ELISA para detectar IgG y los ensayos de Western blot, que tienen alta sensibilidad y especificidad ${ }^{(2-4,8-11)}$.

En Uruguay se disponen de datos epidemiológicos en relación con las geohelmintiasis en niños. Sin embargo, no se dispone de datos de seroprevalencia y menos aun de descripciones de las manifestaciones clínicas de esta enfermedad. Es fundamental el conocimiento de esta zoonosis para fortalecer las medidas de control, prevención, así como de diagnóstico y tratamiento oportuno $^{(12,13)}$.

\section{Objetivo}

Describir las manifestaciones clínicas y de laboratorio de la toxocariasis en menores de 15 años asistidos en una policlínica especializada de un prestador integral privado de salud de Montevideo, entre 2014 y 2018.

\section{Material y método}

Estudio descriptivo, retrospectivo, de los menores de 15 años derivados a la policlínica de infectología de la institución Médica Uruguaya entre el 1 de enero de 2014 y el 31 de diciembre de 2018 con diagnóstico de toxocariasis.

En la confirmación diagnóstica se utilizó la detección de anticuerpos de tipo IgG contra antígenos de excreción/secreción de las larvas de Toxocara canis mediante técnica de ELISA, ensayo por inmunoabsorción ligado a enzimas (Nova Tec ${ }^{\mathbb{B}}$. Esta técnica tiene una sensibilidad de $80 \%$ a $100 \%$ y una especificidad de $90 \%$ a $95 \%{ }^{(14-16)}$.

Se consideraron valores diagnósticos títulos de $\operatorname{IgG}$ mayores a 11 UI NTC (Novatec unidades).

Se analizaron las siguientes variables: edad, sexo, manifestaciones clínicas, motivos de consulta o derivación, hallazgos en el fondo de ojo, recuento leucocitario y de eosinófilos, parasitosis asociadas, tratamiento y evolución.

Todos los niños fueron valorados por oftalmólogo.

Se definió eosinofilia el recuento absolutos de eosinófilos (RAE) mayor a 450 células $/ \mathrm{mm}^{3}$, moderada cuando el RAE es mayor a 1.000-1.500 células $/ \mathrm{mm}^{3}$ y masiva o intensa con RAE $>5.000$ células $/ \mathrm{mm}^{3(14)}$.

La fuente de datos fueron las historias clínicas y la base de datos informatizada del laboratorio.

Las variables cualitativas se expresaron como frecuencias absolutas y relativas; las variables cuantitativas en medidas de tendencia central y rango.

El protocolo contó con la autorización de la dirección técnica de la institución. Se solicitó consentimiento informado a los padres o tutores de los niños. Los datos fueron anonimizados y se resguardó la confidencialidad.

\section{Resultados}

En el período estudiado fueron asistidos en la policlínica de infectología 20 niños con diagnóstico de toxocariasis confirmada por serología.

La media de edad fue 6 años (1-13); 16 de sexo masculino.

Al momento de la consulta, 11 eran asintomáticos y 9 sintomáticos. En relación con las formas sintomáticas, cinco niños presentaron síndrome de LMV y cuatro síndrome de LO.

La media de edad de los niños asintomáticos fue de 5 años, de aquellos con síndrome de LMV visceral 5 años y con síndrome de LO de 9 años.

Los motivos de consulta de los nueve niños sintomáticos fueron: disminución de la agudeza visual, 4; dolor abdominal más hepatomegalia, 2; astenia más hepatome- 
Tabla 1. Características clínicas y de laboratorio en niños con toxocariasis (2014-2018). Montevideo, Uruguay.

\begin{tabular}{|c|c|c|c|}
\hline & Asintomáticos $(n=11)$ & Sindrome LMV $(n=5)$ & Sindrome LO $(n=4)$ \\
\hline Edad años media & 5 & 5 & 9 \\
\hline Sexo (n) Masculino & 9 & 4 & 3 \\
\hline Femenino & 2 & 1 & 1 \\
\hline $\begin{array}{l}\text { Motivo de consulta o derivación a la } \\
\text { policlinica }\end{array}$ & $\begin{array}{l}\text { Eosinofilia (4) } \\
\text { Hábito de pica. Juegos en la tierra, } \\
\text { contacto con perros y gatos (3) } \\
\text { Otras parasitosis (2) } \\
\text { Familiar con toxocariasis (2) }\end{array}$ & $\begin{array}{l}\text { Dolor abdominal, hepatomegalia (2) } \\
\text { Astenia y hepatomegalia (1) } \\
\text { Neumonía, fiebre, hepatomegalia (1) } \\
\text { Sibilancias recurrentes eosinofilia (1) }\end{array}$ & $\begin{array}{l}\text { Disminución de la visión (4) } \\
\text { ) }\end{array}$ \\
\hline Manifestaciones clínicas & & $\begin{array}{l}\text { Hepatomegalia (4) } \\
\text { Astenia marcada (1) } \\
\text { Síndrome Loeffler (2) }\end{array}$ & $\begin{array}{l}\text { Uveítis (2) } \\
\text { Megalopapila (1) } \\
\text { Cicatriz retina (1) }\end{array}$ \\
\hline \multicolumn{4}{|l|}{ Laboratorio } \\
\hline Leucocitos cel/mm³ (rango, media) & $\begin{array}{l}(6.500-10.800) \text { media: } 8.485 \\
(6.200-18.100) \text { media } 10.175\end{array}$ & (9.200-68.300) media: 35.200 & \\
\hline $\mathrm{RAE}^{*}$ (rango, media) & (591-3.392) media 1.395 & $(1.104-40.200)$ media 15.826 & (155-1.748) media 6.421 \\
\hline RAE moderada & 3 & 2 & 1 \\
\hline RAE masiva & 0 & 2 & 0 \\
\hline Hallazgos oftálmicos (n) & 0 & 0 & 4 \\
\hline
\end{tabular}

galia, 1 ; sibilancias recurrentes 1 y neumonía febril más hepatomegalia, 1 (tabla 1).

Con respecto a los hallazgos de laboratorio de los 20 niños, se solicitó hemograma a 16. El rango de leucocitos fue 6.200 a $68.300 / \mathrm{mm}^{3}$ y de RAE 155 a $40.200 \mathrm{cel} / \mathrm{mm}^{3}$. La media del recuento leucocitario fue $15.056 \mathrm{y}$ del RAE 4.727 eosinófilos $/ \mathrm{mm}^{3}$. Presentaban eosinofilia moderada 6 y masiva 2 .

Los niños con síndrome de LO presentaron: dos uveítis posterior, uno megalopapila y un niño ya presentaba una cicatriz en la retina.

Todos los pacientes fueron tratados con albendazol, uno recibió albendazol y mebendazol.

Los pacientes con compromiso ocular y manifestaciones respiratorias recibieron glucocorticoides.

En la tabla 1 se resumen las manifestaciones clínicas y hallazgos de laboratorio de los niños asistidos.

Se constató pérdida de agudeza visual en dos pacientes.

Dos niños presentaron parasitismo asociado por Enterobius vermicularis.

\section{Discusión}

La toxocariasis es una infección parasitaria que se observa fundamentalmente en la niñez, con escaso reconocimiento como un problema de salud pública ${ }^{(2,7,8)}$.
En los países desarrollados oscila entre $2 \%$ y $10 \%{ }^{(3-8)}$. En Uruguay, no hay trabajos publicados de serie de casos. Se menciona la prevalencia en una revisión sistemática realizada en Colombia ${ }^{(3)}$. No es una enfermedad de notificación obligatoria, por lo que hay un subregistro en el número de casos. En nuestro país, la Dra. Hernández y colaboradores realizaron un estudio en el año 2000 de búsqueda de huevos de Toxocara spp en muestras de plazas de Montevideo y encontraron una prevalencia de plazas contaminadas de $52,9 \%$ y de materias fecales de 12,9\%. Los suelos más contaminados fueron los de tierra (78\%), seguidos por los de mezcla $(32 \%)^{(17)}$. También se había estimado una prevalencia en Uruguay de $13,7 \%$ y $33,3 \%$ en autopsias de perros procedentes de la perrera nacional de Montevideo y en $48,3 \%$ de coproparasitarios de perros con dueño ${ }^{(18,19)}$.

Nuestro trabajo describe la primera comunicación de una serie de casos de niños con toxocariasis que se comunica en nuestro medio. A pesar de las limitaciones vinculadas con el diseño retrospectivo y el sesgo de inclusión, por tratarse de un grupo de niños que se asisten en una policlínica especializada, contribuye al conocimiento de las formas de presentación clínica de la enfermedad.

La población estudiada está sesgada, ya que se trata de una policlínica de infectología y no todos los niños 
son derivados. La edad del paciente puede indicar un mayor riesgo de tener toxocariasis clínicamente sintomática. LMV suele ser más frecuente en niños menores de 5 años, por lo cual la media hallada en nuestra seria fue de 5 años y para LO se observa con mayor frecuencia en niños de entre 6 y 14 años, con una media hallada en nuestra serie de 9 años, que coincide con lo descripto en la literatura internacional ${ }^{(20)}$. El sexo masculino predominó en nuestra serie, aunque no se considera un factor de riesgo como el hábito de geofagia o el contacto con perros cachorros ${ }^{(20,21)}$.

En la tabla 1 se puede observar que en los niños asintomáticos $(11 / 20)$ se diagnosticó la infección al detectar factores de riesgo por familiares que padecían la infección, y en otros casos se solicitó serología por la eosinofilia como hallazgo en el hemograma solicitado por otras patologías. En este grupo, 3/11 tenían eosinofilia moderada. De los niños sintomáticos, 4/9 consultaron por alteraciones visuales y dos presentaron pérdida severa de la agudeza visual. Uno de los niños presentó dificultades diagnósticas por hipereosinofilia, descartando enfermedad hematooncológica. En nuestra serie, 4/5 con SLMV presentaron hepatomegalia y $2 / 5$ síntomas respiratorios. Los pacientes con síntomas respiratorios plantearon al inicio dudas diagnósticas en cuanto a la etiología. Dos pacientes presentaron eosinofilias masivas muy elevadas, descartando compromiso pulmonar (fibrosis) y miocárdico (miocarditis eosinofílica). La toxocariasis encubierta es otra forma poco conocida en la literatura, pero que tal vez resulte ser la más frecuente. Este tipo de toxocariasis se caracteriza por tener signos y síntomas inespecíficos y que no entran en la categoría de los clásicos de SLMV o neurotoxocariasis. De los 20 niños asistidos, una niña requirió hospitalización con diagnóstico de síndrome de Loeffler, sibilancias, infiltrados intersticiales difusos pulmonares y un recuento de leucocitos de $68.300 \mathrm{cel} / \mathrm{mm}^{3}$ y de eosinófilos hasta $40.200 \mathrm{cel} / \mathrm{mm}^{3}$. En ninguno de los niños se constató ascaridiasis, ya que este parásito puede dar reacción cruzada antigénica con la técnica de ELISA para toxocariasis.

El diagnóstico etiológico se realiza por pruebas indirectas pues el parásito permanece en forma de larva, no siendo útil el coproparasitario para detectar huevos en las heces. En forma excepcional se puede identificar las larvas en biopsias de los tejidos afectados del paciente o en necropsias ${ }^{(20,21)}$.

Con respecto a la técnica diagnóstica se recomienda la técnica de ELISA para detectar IgG y los ensayos de Western blot, que tienen alta sensibilidad y especificidad, aunque no existan diferencias en ninguno de estos tests entre enfermedad activa o pasada. En nuestra serie se utilizó la técnica ELISA. No se recomienda para el seguimiento la utilización de estas técnicas, ya que perma- nece un título casi constante durante un largo período aun después de haber finalizado el tratamiento del paciente $^{(5)}$. Se estima una sensibilidad y especificidad mayor a $95 \%$ con la técnica utilizada en este estudio, según el manufacturador ${ }^{(5,15)}$. Los casos de falsos negativos son raros y pueden ocurrir en infecciones localizadas leves (como LO) o inmunodeprimidos ${ }^{(20)}$. La detección de IgM no se utiliza, ya que en la fase aguda es difícil realizar el diagnóstico clínico y existen pocos reportes del uso de este anticuerpo ${ }^{(21)}$. Las técnicas de biología molecular se están desarrollando en algunos países, aunque estas técnicas no están estandarizadas para su uso. El seguimiento de los niños se realizó con el recuento absoluto de eosinófilos, en el caso de los niños con compromiso ocular por el oftalmólogo, no se utilizó la serología para el seguimiento, pues se trata de una técnica semicuantitativa.

Con respecto al tratamiento en esta serie todos los pacientes recibieron albendazol y uno albendazol más mebendazol. La duración del tratamiento fue acorde a las recomendaciones para las distintas formas clínicas. Los pacientes asintomáticos fueron tratados con albendazol $400 \mathrm{mg}$ vía oral cada 12 horas por cinco días. Los pacientes con compromiso ocular recibieron, además, corticoides y la paciente hospitalizada requirió corticoides intravenosos. Se recomiendan tratamientos prolongados de dos a cuatro semanas cuando existe compromiso ocular y neurotoxocariasis. Existen controversias respecto al inicio de tratamiento en los pacientes asintomáticos $^{(14)}$.

Las medidas de prevención son importantes en esta enfermedad y debemos ser conscientes de éstas. Lavado de manos correcto, desparasitar a perros y gatos, lavar correctamente las verduras y frutas que se comen crudas, recoger la materia fecal de perros y gatos, y el correcto suministro de agua potable a toda la población ${ }^{(15-21)}$

\section{Conclusiones}

La toxocariasis se puede presentar con síntomas poco específicos. Debido al riesgo del compromiso ocular y de otros órganos en cualquier etapa de la enfermedad sin tener correlación con el título de anticuerpos, parece justificarse el tratamiento antiparasitario en todo los casos. En nuestra serie, si bien la mayoría de los niños fueron asintomáticos, la toxocariasis ocular representó un problema, ya que dos niños tuvieron secuelas severas. Debería realizarse la búsqueda de la infección mediante serología en niños que presenten eosinofilia con y sin síntomas respiratorios o disminución de la agudeza visual causada por lesión intraocular y en niños con factores de riesgo para esta parasitosis. Se requiere sensibili- 
zación y avanzar en el conocimiento de esta patología por las graves consecuencias.

\section{Abstract}

Introduction: human toxocariasis is a widely distributed parasitosis around the world. Its etiological agents are Toxocara canis and Toxocara cati in a smaller proportion.

Objective: to describe the clinical and paraclinical manifestations of toxocariasis in children younger than 15 years old who were assisted in a private comprehensive healthcare provider in Montevideo between 2014 and 2018.

Method: descriptive, retrospective study of children under 15 years old who were referred to the infectology service between January 1, 2014 and December 31, 2018 with a diagnosis of toxocariasis confirmed by ELISA. Variables were age, sex, reason of consultation, clinical manifestations, leukocyte and eosinophils count and associated parasitosis, treatment and evolution.

Results: 20 children were assisted, 11 of them were asymptomatic, 5 evidenced visceral larva migrans (VLM) syndrome and 4 of them presented larva migrans ocular (LMO). 20 patients received albendazon and one of them also received mebendazol. Those whose eyes were compromised also received oral corticoids. Two patients presented loss of visual acuity.

Discussion and conclusions: Most children were asymptomatic, $25 \%$ of them consulted for visual alterations whose sequelae represented a potentially preventable severe health problem. We need to make a greater effort to insist on eye control for children since alterations may be detected, avoiding sequelae with an early diagnosis and the right treatment.

\section{Resumo}

Introdução: a toxocaríase humana é uma parasitose com ampla distribuição em todo o mundo; seus agentes etiológicos são o Toxocara canis e menos frequentemente Toxocara cati.

Objetivo: descrever as características clínicas e de laboratório de toxocaríase em menores de 15 anos atendidos em uma instituição privada de assistência integral de saúde em Montevidéu no período 2014-2018.

Materiais e métodos: estudo descritivo, retrospectivo, dos menores de 15 anos referidos ao ambulatório de infectologia no período 1 de janeiro de 2014 - 31 de dezembro de 2018 com diagnóstico de toxocaríase confirmado por ELISA. Variáveis: idade, sexo, motivo de consulta, manifestações clínicas, contagem de leucócitos e de eosinófilos, parasitoses associadas, tratamento e evolução.
Resultados: vinte crianças foram atendidas sendo: 11 assintomáticos, 5 com síndrome de larva migrans visceral (LMV) e 4 síndrome de síndrome de larva migrans ocular (LO). Todas foram medicadas com albendazol e uma recebeu também mebendazol. Aquelas com compromisso ocular tomaram também corticoides por via oral. Dois pacientes apresentaram perda da acuidade visual.

Discussão e conclusões: a maioria das crianças eram assintomáticas, sendo que um quarto consultou por alterações visuais cujas sequelas representam um problema grave de saúde potencialmente previnivel. Devemos melhorar os esforços para insistir na realização de controle oftalmológico das crianças nos controles de saúde, pois com um diagnóstico precoce e tratamento adequado é possível detectar alterações e evitar sequelas.

\section{Bibliografía}

1. Kimberlin D, Brady MT. Toxocariasis. En: Kimberin D, Brady M, Jackson M, Long S. Red Book: 2018-2021 report of the Committee on Infectious Diseases. 31 ed. Itasca, IL: American Academy of Pediatrics, 2018:808-09.

2. López M, Martín G, Chamorro M, Alonso M. Toxocariasis en niños de una región subtropical. Medicina (B. Aires) 2005; 65(3):226-30.

3. Altcheh J, Nallar M, Conca M, Biancardi M, Freilij H. Toxocariasis: aspectos clínicos y de laboratorio en 54 pacientes. An Pediatr 2003; 58(5):425-31.

4. Guarín Patarroyo C. Situación de la toxocariasis en algunos países de Latinoamérica: revisión sistemática. Bogotá: Universidad Nacional de Colombia, Facultad de Medicina, Instituto de Salud Pública, Departamento de Salud Pública, 2014. Disponible en: www.bdigital.unal.edu.co/44413/1/715569. 2014.pdf. [Consulta: 24 enero 2018].

5. Vázquez Tsuji O, Martínez Barbarosa I, Tay Zavala J, Ruíz Hernández A, Pérez Torres A. Verduras de consumo como probable fuente de infección de toxocara sp. para el hombre. Bol Chil Parasitol 1997; 52(3/):47-50.

6. Vargas C, Torres P, Jercic M, Lobos M, Oyarce A, Miranda $\mathbf{J}$, et al. frequency of anti- toxocara spp. antibodies in individuals attended by the centro de salud familiar and environmental contamination with toxocara canis eggs in dog feces, in the coastal niebla town, Chile. Rev Inst Med Trop Sao Paulo 2016; 58:62.

7. Peña I, Vidal F, del Toro A, Hernández A, Zapata M. Zoonosis parasitarias causadas por perros y gatos, aspecto a considerar en Salud Pública de Cuba. Rev Electrón Vet 2017; 18(10):1-11. Disponible en: https://www.redalyc.org/articulo.oa?id= 63653470002. [Consulta: 14 diciembre 2018].

8. Herskovic P. Larvas migrantes. En: Atias Neghme A, ed. Parasitología clínica. 3 ed. Santiago de Chile: Mediterráneo, 1991:314-8

9. Huapaya P, Espinoza Y, Roldán W, Jiménez S. Toxocariosis humana: ¿problema de salud pública? An Fac Med 2009; 
70(4):283-90 Disponible en: http://www.scielo.org.pe/ pdf/afm/v70n4/a10v70n4.pdf. [Consulta: 14 diciembre 2018].

10. Breña J, Hernández R, Hernández A, Castañeda R, Espinoza Y, Roldán W, et al. Toxocariosis humana en el Perú: aspectos epidemiológicos, clínicos y de laboratorio. Acta Med Per 2011; 28(4):228-36. Disponible en: http://www.scielo.org.pe/ pdf/amp/v28n4/a10.pdf. [Consulta: 21 diciembre 2018].

11. Griffin D, Despommier D. Tissue Nematodes. En: Long S, Prober $\mathrm{Ch}$, Fischer M, eds. Principles and practice of pediatric infectious diseases. 5 ed. Philadelphia: Elsevier, 2018:1381-8.

12. Wiœniewska-Ligier M, WoŸniakowska-Gêsicka T, Sobolewska-Dryjañska J, Markiewicz-JóŸwiak A, Wieczorek M. Analysis of the course and treatment of toxocariasis in children-a long-term observation. Parasitol Res 2012; 110(6):2363-71.

13. Assandri E, Skapino E, Da Rosa D, Alemán A, Acuña A. Anemia, estado nutricional y parasitosis intestinales en niños pertenecientes a hogares vulnerables de Montevideo. Arch Pediatr Urug 2018; 89(2):86-98.

14. Cabrera F, Iturralde A, Lena A, Saavedra M, La Cámera M, García L, et al. Enteroparasitosis en niños de dos Centros de Atención a la Infancia y la Familia (CAIF) del barrio Casavalle, Montevideo. Arch Pediatr Urug 2017; 88(6):315-21.

15. Vickery BP. Eosinófilos. En: Kliegman R, StantonB, St. Geme J, Schor N. Nelson tratado de pediatría. 20 ed. Philadelphia: Elsevier, 2016:1086-9.
16. Yunus M, Tan Farrizam S, Abdul Karim I, Noordin R. A lateral flow rapid test for human toxocariasis developed using three toxocara canis recombinant antigens. Am J Trop Med Hyg 2018; 8(1):32-8.

17. El-Sayed N, Ramadan M. Toxocariasis in children: an update on clinical manifestations, diagnosis, and treatment. J Pediatr Infect Dis 2017; 12(4):222-7.

18. Hernández S, Contera M, Acuña A, Elhordoy D, Vignolo J. Toxocara spp en muestras de suelos y heces de plazas de la ciudad de Montevideo. Rev Patol Trop 2003; 32(1):95-104.

19. Holcman Spector B, Olague G, Couto A. Helmintiasis del perro vagabundo (Canis familiaris) en la ciudad de Montevideo. Rev Urug Patol Clin 1985; 21:67-73.

20. Correa O, Cabrera A. Estudio de relevamiento parasitario de caninos urbanos. En: Primera Jornada de parasitología para estudiantes. Universidad de la República, Facultad de Veterinaria, Departamento de Parasitología. Montevideo, 17 de noviembre de 1998.

21. Roldán W, Espinoza Y, Huapaya P, Jiménez S. Diagnóstico de la toxocariasis humana. Rev Perú Med Exp Salud Pública 2010; 27(4):613-20.

22. Smith H, Holland C, Taylor M, Magnaval J, Schantz P, Maizels R. How common is human toxocariasis? Towards standardizing our knowledge. Trends Parasitol 2009; 25(4):182-8.

\section{Contribución de autores}

Patricia Barrios, https://orcid.org/0000-0002-9199-3188. Participación en todas las etapas del trabajo: concepción, diseño, ejecución, análisis, interpretación de los resultados, redacción, revisión crítica.

Joaquin Mauvezin, https://orcid.org/0000-0002-5982-6910. Participación en todas las etapas del trabajo: concepción, diseño, ejecución, análisis, interpretación de los resultados, redacción, revisión critica.

Yester Basmadjián, https://orcid.org/0000-0003-2783-1499. Diseño, análisis, interpretación de los resultados, redacción y revisión crítica. Beatriz Sayagués, https://orcid.org/0000-0002-2203-7045. Diseño, análisis, redacción y revisión crítica.

Gustavo Giachetto, https://orcid.org/0000-0003-3775-4773. Diseño del trabajo, elaboración de los materiales y métodos, análisis e interpretación de resultados, discusión y conclusiones. 